\title{
ON NET MAPS: EXAMPLES AND NONEXISTENCE RESULTS
}

\author{
EDGAR A. SAENZ
}

\begin{abstract}
A Thurston map is called nearly Euclidean if its local degree at each critical point is 2 and it has exactly four postcritical points. Nearly Euclidean Thurston (NET) maps are simple generalizations of rational Lattès maps. We investigate when such a map has the property that the associated pullback map on Teichmüller space is constant. We also show that no Thurston map of degree 2 has constant pullback map.
\end{abstract}

\section{INTRODUCTION}

Let $S^{2}$ be a topological 2-sphere with a fixed orientation. We use $\mathbb{P}^{1}$ to denote the Riemann sphere. In this paper, all maps $S^{2} \rightarrow S^{2}$ will be orientation preserving. Let $f: S^{2} \rightarrow S^{2}$ be a branched cover, and let $\Omega_{f}$ be the set of its critical points. We define the postcritical set of $f$ to be

$$
P_{f}:=\bigcup_{n>0} f^{\circ n}\left(\Omega_{f}\right)
$$

If $P_{f}$ is finite, we call $f$ a Thurston map. Two Thurston maps $f$ and $g$ are called equivalent iff there exist homeomorphisms $h_{0}:\left(S^{2}, P_{f}\right) \rightarrow\left(S^{2}, P_{g}\right)$ and $h_{1}:\left(S^{2}, P_{f}\right) \rightarrow\left(S^{2}, P_{g}\right)$ for which $h_{0} \circ f=g \circ h_{1}$ and $h_{0}$ is isotopic, rel $P_{f}$, to $h_{1}$. In this case, if $g$ is a rational map we also say that $f$ is realized by $g$.

Suppose $f: S^{2} \rightarrow S^{2}$ is a Thurston map. The orbifold $\mathcal{O}_{f}=\left(S^{2}, \nu_{f}\right)$ associated to $f$ is the topological orbifold with underlying space $S^{2}$ and whose weight function $\nu_{f}(x)$ at $x$ is given by $\nu_{f}(x)=\operatorname{lcm}\left\{n \in \mathbb{Z}^{+}\right.$: there exists a positive integer $m$ such that $f^{\circ m}$ has degree $n$ at some $y \in S^{2}$ with $\left.f^{\circ m}(y)=x\right\}$. Let $\mathcal{T}_{f}$ be the Teichmüller space of $\mathcal{O}_{f}$. We may regard the space $\mathcal{T}_{f}$ as the space of complex structures on $\mathcal{O}_{f}$, up to the equivalence of isotopy fixing $P_{f}$. A complex structure on $\mathcal{O}_{f}$ pulls back under $f$ to a complex structure on $\left(S^{2}, f^{-1}\left(\nu_{f}\right)\right)$, and this extends to a complex structure on $\mathcal{O}_{f}$. In this way we obtain a map $\Sigma_{f}: \mathcal{T}_{f} \rightarrow \mathcal{T}_{f}$. We will refer to $\Sigma_{f}$ as the pullback map induced by $f$.

In [5], Douady and Hubbard, following Thurston, provide necessary and sufficient conditions for a Thurston map to be equivalent to a rational map.

Theorem 1.1 (Thurston). A Thurston map $f$ is equivalent to a rational map if and only if $\Sigma_{f}$ has a fixed point.

One would expect it to be rare (if it happens at all) for a Thurston map $f$ to have the pullback map $\Sigma_{f}$ be constant. In the Buff-Epstein-Koch-Pilgrim paper [2], the authors give an example where the pullback map $\Sigma_{f}$ is constant and characterize when $\Sigma_{f}$ is constant. The example uses a result of McMullen (Proposition 5.1 in [2])

Received by the editors July 4, 2015, and, in revised form, and August 16, 2018.

2010 Mathematics Subject Classification. Primary 37F10, 37F20. 
to construct a Thurston map $f$ with $\Sigma_{f}$ constant by having $\Sigma_{f}$ factor through a trivial Teichmüller space. That result is formulated as follows.

Theorem 1.2 (McMullen). Let $s: \mathbb{P}^{1} \rightarrow \mathbb{P}^{1}$ and $g: \mathbb{P}^{1} \rightarrow \mathbb{P}^{1}$ be rational maps with critical value sets $V_{s}$ and $V_{g}$. Let $A \subset \mathbb{P}^{1}$. Assume $V_{s} \subseteq A$ and $V_{g} \cup g(A) \subseteq s^{-1}(A)$. Then

- $f=g \circ s$ is a Thurston map,

- $V_{g} \cup g\left(V_{s}\right) \subseteq P_{f} \subseteq V_{g} \cup g(A)$, and

- the dimension of the image of $\Sigma_{f}: \operatorname{Teich}\left(\mathbb{P}^{1}, P_{f}\right) \rightarrow \operatorname{Teich}\left(\mathbb{P}^{1}, P_{f}\right)$ is at most $|A|-3$.

We refer to the assumptions of this theorem as McMullen's constant conditions. We showed in 8] that not every Thurston map whose Teichmüller map is constant satisfies McMullen's constant conditions. The Teichmüller map associated to the rational map $f(z)=-\sqrt[3]{2} z\left(z^{3}+2\right) /\left(2 z^{3}+1\right)$ is constant (see Appendix D of [8]). The ramification portrait for this map $f$ is:

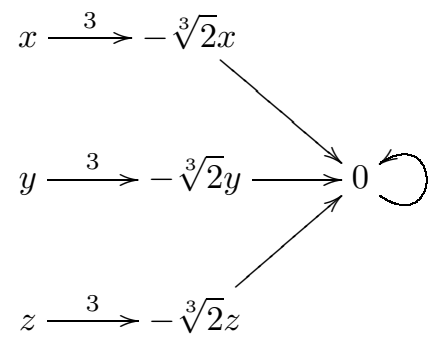

where $x=-1 / 2+\sqrt{3} i / 2, y=-1 / 2-\sqrt{3} i / 2$, and $z=1$. However, $f$ cannot be written as the composition of two maps of degree greater than one because $\operatorname{deg}(f)=4$ and the local degree of $f$ at every critical point is 3 .

Other examples of Thurston maps whose induced maps on Teichmüller space are constant and that do not satisfy McMullen's constant conditions can be found in the class of topological branched coverings called Nearly Euclidean Thurston maps (NET maps). A Thurston map $f: S^{2} \rightarrow S^{2}$ is a NET map if its local degree at each of its critical points is 2 and it has exactly four postcritical points.

Any NET map $f$ admits what we call a NET map presentation. That is, given a NET map $f$, we can associate to $f$ a quintuple $\left(\Lambda_{1}, \Phi, R, h_{R}, h\right)$ where $\Lambda_{1}$ is a lattice in $\mathbb{R}^{2}$ (see Section 3 ). This combinatorial data allows us to algorithmically compute the number of essential components in the preimage of an essential simple closed curve contained in $S^{2} \backslash P_{f}$. The following theorem can be found in [4].

Theorem 1.3. Let $\Lambda_{2}=\mathbb{Z}^{2}$, and let $\delta$ be an essential simple closed curve in $S^{2} \backslash P_{f}$ with slope $p / q$ with respect to the canonical basis of $\Lambda_{2}$. Let $\lambda=(q, p) \in \Lambda_{2}$. Since $p$ and $q$ are relatively prime, there exists $\mu \in \Lambda_{2}$ such that $\lambda$ and $\mu$ form a basis for $\Lambda_{2}$. Let $c_{1}, c_{2}, c_{3}, c_{4}$ be the coset numbers for $H=p_{1}^{-1}\left(P_{f}\right) \subset \Lambda_{2} / 2 \Lambda_{1}$ relative to $\lambda$ and $\mu$. Then, the number of essential components in $f^{-1}(\delta)$ is $c_{3}-c_{2}$.

This result and Theorem 5.1 of [2] imply that the Teichmüller map of a NET map $f$ is constant if and only if $c_{2}=c_{3}$ for every choice of $\lambda$ and $\mu$. In [4], J. Cannon et al. use this constraint on $c_{2}$ and $c_{3}$ to define the concept of a nonseparating set (a set that never separates $c_{2}$ from $c_{3}$ ), then the authors use the definition of a nonseparating set to provide an algebraic characterization of those NET maps whose 
induced maps on Teichmüller space are constant. This characterization reduces to the existence of a nonseparating set within the finite Abelian group $\Lambda_{2} / 2 \Lambda_{1}$.

We are particularly interested in the existence of nonseparating sets contained in finite Abelian groups. Our main result is focused on this purely algebraic problem.

Theorem 1.4 (Main Theorem). Let $A$ be a finite Abelian group generated by two elements such that $A / 2 A \cong \mathbb{Z} / 2 \mathbb{Z} \oplus \mathbb{Z} / 2 \mathbb{Z}$. If $|A|=4 p_{1}^{k_{1}} p_{2}^{k_{2}} \cdots p_{n}^{k_{n}}$ with $p_{i}$ prime, $p_{i} \geq 13$, and $|k|=k_{1}+k_{2}+\cdots+k_{n} \geq 1$, then $A$ does not contain a nonseparating subset.

As a consequence of this, if $n=p_{1}^{k_{1}} p_{2}^{k_{2}} \cdots p_{n}^{k_{n}}$ where each $p_{i}$ is at least 13 , then there does not exist a NET map with degree $n$ whose Teichmüller map is constant.

This paper is organized as follows. Section 2 sets notation, reviews results, and provides new algebraic properties related to nonseparating sets which will be needed in the sequel. Section 3 introduces NET maps, reviews basic facts, and applies the theory of Section 2 in the construction of two examples of NET maps whose Teichmüller maps are constant. One of these examples does not satisfy McMullen's constant conditions. In Section 4 we investigate when the induced pullback map on Teichmüller space of NET maps cannot be constant. In Section 5 we show that no Thurston map of degree 2 has constant pullback map. The proof of Corollary 5.3. in the case $\left|P_{f}\right|=4$, gives a new proof of Theorem 4.1 .

\section{Coset numbers And NOnSeparating Sets}

In this section, we first review the definitions and facts on coset numbers and nonseparating sets. Then we prove the converse of Lemma 2.4 and a technical lemma relevant in the proof of the main result.

Let $A$ be a finite abelian group. Let $H$ be a subset of $A$ which is the disjoint union of four pairs $\left\{ \pm h_{1}\right\},\left\{ \pm h_{2}\right\},\left\{ \pm h_{3}\right\},\left\{ \pm h_{4}\right\}$. (It is possible that $h_{i}=-h_{i}$.) Let $B$ be a subgroup such that $A / B$ is cyclic, and let $a \in A$ so that $a+B$ generates $A / B$. Let $n$ be the order of $A / B$. For each $k \in\{1,2,3,4\}$ there exists a unique integer $c$ with $0 \leq c \leq n / 2$ such that $(c a+B) \cap\left\{ \pm h_{k}\right\} \neq \emptyset$. Let $c_{1}, c_{2}, c_{3}, c_{4}$ be these four integers ordered so that $0 \leq c_{1} \leq c_{2} \leq c_{3} \leq c_{4}$. These four numbers are called coset numbers for $H$ relative to $B$ and the generator $a+B$ of $A / B$.

Let $A$ be a finite Abelian group. A subset $H$ of $A$ is called nonseparating if and only if it satisfies the following conditions:

- $H$ is a disjoint union of the form $H=H_{1} \coprod H_{2} \amalg H_{3} \coprod H_{4}$, where each $H_{i}$ has the form $H_{i}=\left\{ \pm h_{i}\right\}$. (It is possible that $h_{i}=-h_{i}$.)

- Let $B$ be a cyclic subgroup of $A$ such that $A / B$ is cyclic. Let $c_{1}, c_{2}, c_{3}, c_{4}$ be the coset numbers for $H$ relative to $B$ and some generator of $A / B$. The main condition is that $c_{2}=c_{3}$ for every such choice of $B$ and generator of $A / B$.

Example 2.1. Let $A=\mathbb{Z} / 3 \mathbb{Z} \oplus \mathbb{Z} / 3 \mathbb{Z}$. The subset $H=A \backslash\{(0,0)\}$ is a nonseparating subset of $A$. In fact, let $B$ be a cyclic subgroup of $A$ so that $A / B$ is cyclic. Then $B \cong A / B \cong \mathbb{Z} / 3 \mathbb{Z}$. Given a generator $a+B$ of $A / B$ we have only three cosets: $B, a+B$ and $2 a+B$. It is obvious that $B$ contains exactly one pair of mutually inverse elements of order 3 . So $c_{1}=0$ and $c_{2}=c_{3}=c_{4}=1$.

Example 2.2. Let $k$ be an integer with $k \geq 3$. Let $A=\mathbb{Z} / 2^{k} \mathbb{Z} \oplus \mathbb{Z} / 2 \mathbb{Z}$, and let $H=\left\{ \pm(1,0), \pm\left(2^{k-2}, 0\right), \pm\left(2^{k-2}, 1\right), \pm\left(2^{k-1}-1,0\right)\right\}$. We show that $H$ is a 
nonseparating subset of $A$. Let $B$ be a cyclic subgroup of $A$ such that $A / B$ is cyclic. Then either $|B|=2^{k}$ or $|B|=2$. First suppose that $|B|=2^{k}$. Then $A / B \cong \mathbb{Z} / 2 \mathbb{Z}$. Given a generator $a+B$ of $A / B$ we have only two cosets: $B$ and $a+B$. In this case to show that $H$ does not separate $c_{2}$ from $c_{3}$ it suffices to prove that $B$ does not contain exactly two elements of $H$. If $(1,0) \in B$, then $\left(2^{k-2}, 0\right)$ and $\left(2^{k-2}-1,0\right) \in B$. The same is true if $\left(2^{k-1}-1,0\right) \in B$. So if $B$ contains exactly two elements of $H$, then these elements are $\left(2^{k-2}, 0\right)$ and $\left(2^{k-2}, 1\right)$. But then $(0,1) \in B$. This is impossible.

Now suppose that $|B|=2$. Then either $B=\langle(0,1)\rangle$ or $B=\left\langle\left(2^{k-1}, 1\right)\right\rangle$. Let $a \in A$ such that $a+B$ generates $A / B$. The first component of $a$ has the form $4 r \pm 1$ for some integer $r$. Hence $2^{k-2} a+B= \pm\left(2^{k-2}, 0\right)+B$, and so the coset number of $\pm\left(2^{k-2}, 0\right)$ is $2^{k-2}$. Similarly, one verifies that the coset number of $\pm\left(2^{k-2}, 1\right)$ is $2^{k-2}$. Let $m$ be the integer in $\left\{0, \ldots, 2^{k-1}\right\}$ such that $m(a+B)= \pm(1,0)+B$. Then $\left(2^{k-1}-m\right)(a+B)= \pm\left(2^{k-1}-1,0\right)+B$. So if $m$ is the coset number of $\pm(1,0)$, then $2^{k-1}-m$ is the coset number of $\pm\left(2^{k-1}-1,0\right)$. Thus, $\left\{c_{1}, c_{4}\right\}=\left\{m, 2^{k-1}-m\right\}$ and $c_{2}=c_{3}=2^{k-2}$. This proves that $H$ is a nonseparating subset of $A$.

The next two lemmas provide ways to produce nonseparating subsets from known ones. For details of the proofs, see Section 10 of 4 .

Lemma 2.3. Let $A$ be a finite Abelian group, and let $H$ be a nonseparating subset of $A$. If $\varphi: A \rightarrow A$ is a group automorphism and $\tau$ is an element of $A$ with $2 \tau=0$, then $\varphi(H)+\tau$ is a nonseparating subset of $A$.

Lemma 2.4. If $A$ is a finite Abelian group and if $A^{\prime}$ is a subgroup of $A$, then every subset of $A^{\prime}$ which is nonseparating for $A^{\prime}$ is nonseparating for $A$.

Example 2.5. Let $A=\mathbb{Z} / 4 \mathbb{Z} \oplus \mathbb{Z} / 2 \mathbb{Z}$. The set $H=\{(0,0), \pm(1,0), \pm(1,1), \pm(2,0)\}$ is a nonseparating subset of $A$. For details of the proof see Example 10.3 of [4]. By Lemma 2.4, $H^{\prime}=\{(0,0), \pm(1,0), \pm(1,2),(2,0)\}$ is a nonseparating subset of $\mathbb{Z} / 4 \mathbb{Z} \oplus \mathbb{Z} / 4 \mathbb{Z}$.

The next lemma shows the converse of Lemma 2.4. For additional details of the proof, see Appendix A.

Lemma 2.6. Let $A$ be a finite Abelian group generated by two elements, and let $A^{\prime}$ be a subgroup of $A$. If $H$ is a subset of $A^{\prime}$ which is nonseparating for $A$, then $H$ is nonseparating for $A^{\prime}$.

Proof. Let $B^{\prime}$ be a cyclic subgroup of $A^{\prime}$ such that $A^{\prime} / B^{\prime}$ is cyclic. Let $a^{\prime}$ be an element of $A^{\prime}$ such that $a^{\prime}+B^{\prime}$ generates $A^{\prime} / B^{\prime}$. By Proposition A.4, there exists $B$ a subgroup of $A$ such that $A / B$ is cyclic and $A^{\prime} \cap B=B^{\prime}$. Let $n$ be the order of $A / B$, and let $m$ be the order of $a^{\prime}+B \in A / B$. By Proposition A.1, there exists an element $a$ in $A$ such that $a+B$ generates $A / B$ and $(n / m)(a+B)=a^{\prime}+B$. Let $0 \leq c_{1} \leq c_{2} \leq c_{3} \leq c_{4} \leq(1 / 2)\left|A^{\prime} / B^{\prime}\right|$ be the coset numbers for $H$ relative to $B^{\prime}$ and the generator $a^{\prime}+B^{\prime}$ of $A^{\prime} / B^{\prime}$. Since $a^{\prime} \in A^{\prime}$ and $m a^{\prime} \in B, m a^{\prime} \in B^{\prime}$. So $\left|A^{\prime} / B^{\prime}\right|$ divides $m$. This yields $0 \leq n c_{1} / m \leq n c_{2} / m \leq n c_{3} / m \leq n c_{4} / m \leq n / 2$. Since $c_{i}\left(a^{\prime}+\right.$ $\left.B^{\prime}\right) \subseteq c_{i}\left(a^{\prime}+B\right)=\left(n c_{i} / m\right)(a+B)$, it follows that $n c_{1} / m, n c_{2} / m, n c_{3} / m, n c_{4} / m$ are the coset numbers for $H$ relative to $B$ and $a+B$. Hence $n c_{2} / m=n c_{3} / m$, and so $c_{2}=c_{3}$.

Let $A$ be a finite Abelian group. Because of Lemma 2.3 we define an equivalence relation $\sim$ on the collection of nonseparating subsets of $A$ as follows. 
Definition 2.7. Let $H_{1}, H_{2}$ be two nonseparating subsets of $A$. We say that $H_{1}$ is related to $H_{2}$ and write $H_{1} \sim H_{2}$ if and only if there exists $\varphi$ an automorphism of $A$ and an element $\tau \in A$ with $2 \tau=0$ such that $H_{2}=\varphi\left(H_{1}\right)+\tau$. The equivalence classes of this equivalence relation are called Hurwitz classes of nonseparating subsets.

Remark 2.8. Let $A, H$ be as in Example 2.2. The group $A$ contains the subgroup $\left\langle\left(2^{k-2}, 0\right)\right\rangle \oplus \mathbb{Z} / 2 \mathbb{Z}$ which is isomorphic to $\mathbb{Z} / 4 \mathbb{Z} \oplus \mathbb{Z} / 2 \mathbb{Z}$. By Lemma 2.4 and Example 2.5 above, $H^{\prime}=\left\{(0,0), \pm\left(2^{k-2}, 0\right), \pm\left(2^{k-1}, 0\right), \pm\left(2^{k-2}, 1\right)\right\}$ is also a nonseparating subset of $A$. If there were an element $\tau$ in $A$ with $2 \tau=0$ and an automorphism $\varphi$ of $A$ such that $H=\varphi\left(H^{\prime}\right)+\tau$, then $\tau=\varphi(0)+\tau$ would be an element of $H$. However, $H$ contains no element of order less than 4. So $H$ and $H^{\prime}$ are representatives of distinct Hurwitz classes of nonseparating subsets of $A$.

Remark 2.9. With the assistance of a computer program W. Parry has verified that there are three Hurwitz classes of nonseparating subsets in $\mathbb{Z} / 4 \mathbb{Z} \oplus \mathbb{Z} / 4 \mathbb{Z}$. Here are representatives for them:

$$
\begin{gathered}
H_{1}=\{(0,0), \pm(1,0), \pm(1,2),(2,0)\}, \\
H_{2}=\{ \pm(1,0), \pm(0,1), \pm(2,1), \pm(1,2)\}, \\
H_{3}=\{ \pm(1,0), \pm(0,1), \pm(1,1), \pm(3,1)\} .
\end{gathered}
$$

A detailed proof of this fact can be found in Appendix B.

We now state and prove a lemma that will be used in the proof of Theorem 4.5.

Lemma 2.10. Let $a$ and $b$ be odd positive integers such that $a \mid b$ and $a>1$. Let $A=\mathbb{Z} / 2 \mathbb{Z} \oplus \mathbb{Z} / 2 \mathbb{Z} \oplus \mathbb{Z} / a \mathbb{Z} \oplus \mathbb{Z} / b \mathbb{Z}$, and let $\phi: A \rightarrow \mathbb{Z} / a \mathbb{Z} \oplus \mathbb{Z} / b \mathbb{Z}$ be the canonical projection. Suppose that $A$ contains a nonseparating subset $H=\coprod_{i=1}^{4}\left\{ \pm h_{i}\right\}$. Let $D=\left\{\phi\left(h_{i}\right) \pm \phi\left(h_{j}\right): i, j \in\{1,2,3,4\}\right.$ with $\left.i<j\right\}$. Assume that there exists a cyclic subgroup $G$ of $\mathbb{Z} / a \mathbb{Z} \oplus \mathbb{Z} / b \mathbb{Z}$ such that $G \cap D \subseteq\{0\}$ and $(\mathbb{Z} / a \mathbb{Z} \oplus \mathbb{Z} / b \mathbb{Z}) / G$ is cyclic. If $\mathbb{Z} / a \mathbb{Z} \oplus \mathbb{Z} / b \mathbb{Z}=\langle\phi(H)\rangle$, then we may assume that $\left\langle\phi\left(h_{1}\right), \phi\left(h_{2}\right)\right\rangle=\mathbb{Z} / a \mathbb{Z} \oplus \mathbb{Z} / b \mathbb{Z}$ and $h_{2}, h_{3}$, and $h_{4}$ all differ by an element of order 2 .

Proof. Define the following three cyclic subgroups of $A$ :

- $E_{(1,0)}=\langle(1,0)\rangle \oplus G$,

- $E_{(0,1)}=\langle(0,1)\rangle \oplus G$,

- $E_{(1,1)}=\langle(1,1)\rangle \oplus G$.

The quotient groups $A / E_{(1,0)}, A / E_{(0,1)}$, and $A / E_{(1,1)}$ are cyclic. Let $x_{i j}+E_{(i, j)}$ be a generator of $A / E_{(i, j)}$. We denote the second coset number for $H$ relative to $E_{(i, j)}$ and the generator $x_{i, j}+E_{(i, j)}$ by $c_{i, j}$.

Since $H$ is a nonseparating subset of $A$, without loss of generality we may assume that $h_{2}$ and $h_{3}$ are elements of the coset $c_{1,0} x_{1,0}+E_{(1,0)}$. Then $\phi\left(h_{2}\right)-\phi\left(h_{3}\right) \in G$. So, $\phi\left(h_{2}\right)=\phi\left(h_{3}\right)$ and $h_{2}-h_{3}=(1,0,0,0)$.

We show that $\left\{ \pm h_{2}, \pm h_{3}\right\} \subset c_{0,1} x_{0,1}+E_{(0,1)}$ cannot occur. Proceed by contradiction. If $\left\{h_{2}, h_{3}\right\} \subset c_{0,1} x_{0,1}+E_{(0,1)}$ or $\left\{-h_{2},-h_{3}\right\} \subset c_{0,1} x_{0,1}+E_{(0,1)}$, then $h_{2}-h_{3}=(0,1,0,0)$, which is impossible. If $\left\{h_{2},-h_{3}\right\} \subset c_{0,1} x_{0,1}+E_{(0,1)}$ or $\left\{-h_{2}, h_{3}\right\} \subset c_{0,1} x_{0,1}+E_{(0,1)}$, then $h_{2}+h_{3}=(0,1,0,0)$, and so $2 h_{2}=(1,1,0,0)$, which yields a contradiction. Similarly, $\left\{ \pm h_{2}, \pm h_{3}\right\} \subset c_{1,1} x_{1,1}+E_{(1,1)}$ cannot occur.

We now show that $\left\{ \pm h_{1}, \pm h_{4}\right\} \subset c_{0,1} x_{0,1}+E_{(0,1)}$ cannot occur. Relabeling, if necessary, it suffices to show that $\left\{h_{1}, h_{4}\right\} \subset c_{0,1} x_{0,1}+E_{(0,1)}$ cannot occur. Proceed 
by contradiction. Suppose that $\left\{h_{1}, h_{4}\right\} \subset c_{0,1} x_{0,1}+E_{(0,1)}$. The above argument shows that $\phi\left(h_{1}\right)=\phi\left(h_{4}\right)$ and that $\left\{ \pm h_{1}, \pm h_{4}\right\} \subset c_{1,1} x_{1,1}+E_{(1,1)}$ cannot occur. Then one of the following sixteen inclusions must hold:

- $\left\{ \pm h_{1}, \pm h_{2}\right\} \subset c_{1,1} x_{1,1}+E_{(1,1)}$,

- $\left\{ \pm h_{1}, \pm h_{3}\right\} \subset c_{1,1} x_{1,1}+E_{(1,1)}$,
- $\left\{ \pm h_{4}, \pm h_{2}\right\} \subset c_{1,1} x_{1,1}+E_{(1,1)}$,

- $\left\{ \pm h_{4}, \pm h_{3}\right\} \subset c_{1,1} x_{1,1}+E_{(1,1)}$.

However, each of them would imply that $\langle\phi(H)\rangle=\left\langle\phi\left(h_{1}\right)\right\rangle$. Since $\mathbb{Z} / a \mathbb{Z} \oplus \mathbb{Z} / b \mathbb{Z}=$ $\langle\phi(H)\rangle$, none of these inclusions occur. So, $\left\{ \pm h_{1}, \pm h_{4}\right\} \subset c_{0,1} x_{0,1}+E_{(0,1)}$ cannot occur.

Now, we may assume that either $\left\{h_{3}, h_{4}\right\}$ or $\left\{-h_{3}, h_{4}\right\}$ is a subset of the coset $c_{0,1} x_{0,1}+E_{(0,1)}$. If $\left\{h_{3}, h_{4}\right\} \subset c_{0,1} x_{0,1}+E_{(0,1)}$, then $h_{3}-h_{4}=(0,1,0,0)$. Hence $\phi\left(h_{2}\right)=\phi\left(h_{3}\right)=\phi\left(h_{4}\right)$ and the lemma follows. If $\left\{-h_{3}, h_{4}\right\} \subset c_{0,1} x_{0,1}+E_{(0,1)}$, then $h_{3}+h_{4}=(0,1,0,0)$. In this case, $\phi\left(h_{2}\right)=\phi\left(h_{3}\right)=-\phi\left(h_{4}\right)$. Relabeling $h_{4}$ by $-h_{4}$, the lemma follows.

\section{NET MAPs: PRELIMINARIES AND EXAMPleS}

In this section we briefly review some definitions and properties of NET maps. We refer the reader to Section 1 in 4 for more details.

Definition 3.1. A Thurston map $f: S^{2} \rightarrow S^{2}$ is called Euclidean if its local degree at each of its critical points is 2 , it has at most four postcritical points, and none of them is critical.

Definition 3.2. A Thurston map $f: S^{2} \rightarrow S^{2}$ is called nearly Euclidean (NET) if its local degree at each of its critical points is 2 and it has exactly four postcritical points.

From Lemma 1.3 of [4, it follows that every Euclidean Thurston map is nearly Euclidean, and every NET map $f$ has the property that $f^{-1}\left(P_{f}\right)$ contains exactly four points which are not critical points; $f$ is Euclidean if and only if these four points are precisely the points of $P_{f}$. The next theorem shows that NET maps lift to maps of tori. The proof of the theorem and the following description can be found in Section 1 of [4].

Theorem 3.3. Let $f$ be a Thurston map. Then $f$ is nearly Euclidean if and only if there exist branched covering maps $p_{1}: T_{1} \rightarrow S^{2}$ and $p_{2}: T_{2} \rightarrow S^{2}$ with degree 2 from the tori $T_{1}$ and $T_{2}$ to $S^{2}$ such that the set of branch of $p_{2}$ is $P_{f}$ and there exists a continuous map $\tilde{f}: T_{1} \rightarrow T_{2}$ such that $p_{2} \circ \tilde{f}=f \circ p_{1}$. If $f$ is nearly Euclidean, then $f$ is Euclidean if and only if the set of branched points of $p_{1}$ is $P_{f}$.

Let $f: S^{2} \rightarrow S^{2}$ be a NET map. Let $p_{1}: T_{1} \rightarrow S^{2}, p_{2}: T_{2} \rightarrow S^{2}$, and $\tilde{f}: T_{1} \rightarrow T_{2}$ as in Theorem 3.3 such that $p_{2} \circ \tilde{f}=f \circ p_{1}$. For $j \in\{1,2\}$, let $P_{j}(f) \subset S^{2}$ be the set of branched points of $p_{j}$ and let $q_{j}: \mathbb{R}^{2} \rightarrow T_{j}$ be a universal covering map. The map $p_{j} \circ q_{j}: \mathbb{R}^{2} \rightarrow S^{2}$ is a regular branched covering map whose local degree at every ramification point is 2 . Let $\Gamma_{j}$ and $\Lambda_{j}$ be the set of deck transformations and the set of ramification points of $p_{j} \circ q_{j}$. We can choose $q_{j}$ so that $\Gamma_{j}$ is generated by the set of all Euclidean rotations of order 2 about the points of $\Lambda_{j}$. We may, and do, normalize so that $0 \in \Lambda_{j}$. Hence $\Lambda_{j}$ is a lattice in $\mathbb{R}^{2}$ and the elements of $\Gamma_{j}$ are the maps of the form $x \mapsto 2 \lambda \pm x$ for some $\lambda \in \Lambda_{j}$. 
The map $\tilde{f} \circ q_{1}$ lifts to a continuous map $\hat{f}: \mathbb{R}^{2} \rightarrow \mathbb{R}^{2}$ such that $q_{2} \circ \hat{f}=\tilde{f} \circ q_{1}$. Since $\tilde{f} \circ q_{1}$ is a covering map, the map $\hat{f}$ is as well. Hence, $\hat{f}$ is a homeomorphism. We replace $q_{1}$ by $q_{1} \circ \hat{f}^{-1}$. In this case, $\tilde{f}$ lifts to the identity map. Thus, $\Lambda_{1} \subseteq \Lambda_{2}$ and $\Gamma_{1} \subseteq \Gamma_{2}$. So we obtain the standard commutative diagram

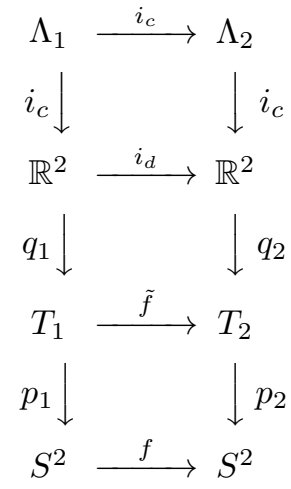

where $i_{d}$ is the identity map and the maps from $\Lambda_{1}$ and $\Lambda_{2}$ are inclusion maps.

The group $\Gamma_{j}$ contains the group of deck transformations of $q_{j}$. It is the subgroup with index 2 consisting of translations of the form $x \mapsto 2 \lambda+x$ with $\lambda \in \Lambda_{j}$. So we can identify $T_{j}$ with $\mathbb{R}^{2} / 2 \Lambda_{j}$. The standard commutative diagram implies that $\mathbb{R}^{2} / \Gamma_{1}$ and $\mathbb{R}^{2} / \Gamma_{2}$ are both identified with $S^{2}$. Thus there is an identification map $\phi: \mathbb{R}^{2} / \Gamma_{2} \rightarrow \mathbb{R}^{2} / \Gamma_{1}$. To evaluate $f$ at some point $x$, we view $x$ as an element of $\mathbb{R}^{2} / \Gamma_{1}$. We lift it to $\mathbb{R}^{2}$, then project it to $\mathbb{R}^{2} / \Gamma_{2}$, and then apply the identification map $\phi$ to obtain $f(x)$. For Euclidean NET maps the identification map $\phi$ can be obtained by using $\Phi: \mathbb{R}^{2} \rightarrow \mathbb{R}^{2}$ an affine automorphism such that $\Phi\left(\Lambda_{2}\right)=\Lambda_{1}$.

The following theorem shows that a NET map $f$ can be obtained by taking a Euclidean Thurston map $g$ and postcomposing it by a homeomorphism $h$ that satisfies $h\left(P_{g}\right) \subseteq g^{-1}\left(P_{g}\right)$, subject to the constraint that the composition $f=h \circ g$ has four postcritical points. More precisely, we have the following.

\section{Theorem 3.4.}

(1) If $g: S^{2} \rightarrow S^{2}$ is a NET map and $h: S^{2} \rightarrow S^{2}$ is an orientation-preserving homeomorphism such that $h\left(P_{g}\right) \subseteq g^{-1}\left(P_{g}\right)$, then $f=h \circ g$ is a NET map if it has at least four post critical points.

(2) Let $f$ be a NET map with $P_{1}=P_{1}(f)$ and $P_{2}=P_{2}(f)$. Let $h: S^{2} \rightarrow S^{2}$ be an orientation-preserving homeomorphism with $h\left(P_{1}\right)=P_{2}$. Then $f=h \circ g$, where $g: S^{2} \rightarrow S^{2}$ is a Euclidean Thurston map with $P_{g}=P_{1}$ and $P_{2} \subseteq g^{-1}\left(P_{g}\right)$, so that $h\left(P_{g}\right) \subseteq g^{-1}\left(P_{g}\right)$.

Combining Theorem 3.3, the description of the standard commutative diagram given above, and Theorem 3.4 each NET map can be constructed as follows. Let $\Lambda_{2}$ be the lattice generated by $(1,0)$ and $(0,1)$. Fix a quintuple $\left(\Lambda_{1}, \Phi, R, h_{R}, h\right)$, where

- $\Lambda_{1}$ is a sublattice of $\Lambda_{2}$ of covolume greater than one. Let $\Gamma_{1}, \Gamma_{2}$ be the groups generated by rotations of order 2 about elements of $\Lambda_{1}, \Lambda_{2}$ so that $S_{1}^{2}:=\mathbb{R}^{2} / \Gamma_{1}, S_{2}^{2}:=\mathbb{R}^{2} / \Gamma_{2}$ are spheres. For $j \in\{1,2\}$, let $\pi_{j}: \mathbb{R}^{2} \rightarrow S_{j}^{2}$ be the canonical quotient map. Since $\Lambda_{1}<\Lambda_{2}$, we have $\Gamma_{1}<\Gamma_{2}$. So the identity map $i: \mathbb{R}^{2} \rightarrow \mathbb{R}^{2}$ induces a map $\bar{i}: S_{1}^{2} \rightarrow S_{2}^{2}$ such that $\bar{i} \circ \pi_{1}=\pi_{2}$. The map $\bar{i}$ is a branched covering map of degree $d:=\left[\Lambda_{2}: \Lambda_{1}\right]$. 
- For $j \in\{1,2\}$, set $T_{j}:=\mathbb{R}^{2} / 2 \Lambda_{j}$, and let $p_{j}: T_{j} \rightarrow S_{j}^{2}$ be branched covering maps of degree 2. Let $P_{j}=\Lambda_{j} / \Gamma_{j}$ be the set of branched points of $p_{j}$. Note that $\left|P_{j}\right|=4$.

- $\Phi:\left(\mathbb{R}^{2}, \Lambda_{2}\right) \rightarrow\left(\mathbb{R}^{2}, \Lambda_{1}\right)$ is an affine map of the form $\Phi(x)=L x+b$ where $b \in \Lambda_{1}$ and $L$ is a $2 \times 2$ matrix over $\mathbb{Z}$ of determinant greater than one.

- $R \subset \Lambda_{2} / \Gamma_{1} \subset S_{1}^{2}$ is a set of four points.

- $h_{R}: P_{1} \rightarrow R$ is a bijection.

- $h:\left(S_{1}^{2}, P_{1}\right) \rightarrow\left(S_{1}^{2}, R\right)$ is an orientation-preserving homeomorphism which is an extension of $h_{R}$ :

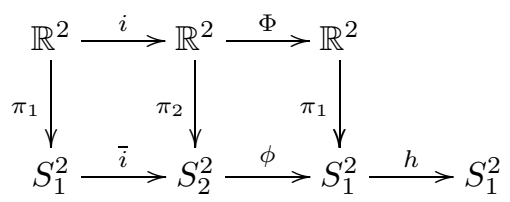

The affine map $\Phi$ descends to a homeomorphism $\phi: S_{2}^{2} \rightarrow S_{1}^{2}$ so that the composition $g=\phi \circ \bar{i}$ is a Euclidean Thurston map such that $P_{g}=P_{1}$. Since $h\left(P_{g}\right)=h\left(P_{1}\right)=R$, it turns out that $f:=h \circ g$ is a Thurston map such that $P_{f} \subset R$. If $V_{g}=P_{1}$ (which is always true if $\operatorname{deg}(g)=3$ or $\operatorname{deg}(g) \geq 5$ ), then $P_{f}=R$ and we get a NET map by this process. Thus, given a nearly Euclidean Thurston map $f$, we can always associate to $f$ a quintuple $\left(\Lambda_{1}, \Phi, R, h_{R}, h\right)$ and a commutative diagram as above where $f=h \circ g=h \circ(\phi \circ \bar{i})$.

Under these settings, in [4] J. Cannon et al. proved the following result.

Theorem 3.5. Let $f$ be a NET, and let $p_{1}, \Lambda_{1}, \Lambda_{2}$ be as above. Then the Teichmüller map of $f$ is constant if and only if $p_{1}^{-1}\left(P_{f}\right)$ is a nonseparating subset of $\Lambda_{2} / 2 \Lambda_{1}$.

So, in order to construct NET maps whose Teichmüller maps are constant, we may consider the following steps:

1. Let $\Lambda_{2}$ be the lattice generated by $(1,0)$ and $(0,1)$.

2. Construct a finite Abelian group $A$ generated by two elements with $A / 2 A \cong$ $\mathbb{Z} / 2 \mathbb{Z} \oplus \mathbb{Z} / 2 \mathbb{Z}$ such that $A$ has a nonseparating subset $H$.

3. Construct a lattice $\Lambda_{1}$ such that $\Lambda_{1}<\Lambda_{2}$ for which $\Lambda_{2} / 2 \Lambda_{1} \cong A$.

4. Construct an isomorphism $\Phi$ from $\Lambda_{2}$ to $\Lambda_{1}$, which in effect produces a Euclidean Thurston map $g$ corresponding to $\Lambda_{1}$ and $\Lambda_{2}$. That is, $g=\phi \circ \bar{i}$.

5. Construct an orientation-preserving homeomorphism $h: S_{1}^{2} \rightarrow S_{1}^{2}$ such that $h\left(P_{g}\right)=p_{1}(H)$. Here $p_{1}: T_{1} \rightarrow S_{1}^{2}$.

6. Set $f:=h \circ g$. By Theorem 3.4 if $f$ has four postcritical points, then it is a NET map. In that case, $P_{f}=h\left(P_{g}\right)$, and so $H=p_{1}^{-1}\left(P_{f}\right)$. Then, by Theorem 3.5, the Teichmüller map of $f$ is constant. Since $\left|\Lambda_{2} / \Lambda_{1}\right|=\operatorname{deg}(f)$, we have $|A|=4 \operatorname{deg}(f)$.

In [4, J. Cannon et al. prove a general existence theorem. If $d$ is an integer with $d>2$ such that $d$ is divisible by either 2 or 9 , then there exists a NET map with degree $d$ whose Teichmüller pullback map is constant. In particular, it is possible to construct NET maps with odd degree and a constant Teichmüller map.

Lemma 3.6. Let $s: S^{2} \rightarrow S^{2}$ be an orientation-preserving branched covering map such that $\operatorname{deg}(s, x)=2$ for every $x \in \Omega_{s}$. If $\left|V_{s}\right| \leq 3$, then $\operatorname{deg}(s)=2$ or $\operatorname{deg}(s)=4$. 
Proof. If $\operatorname{deg}(s)=3$, the preimage under $s$ of every element of $V_{s}$ contains three points counting multiplicity and no such preimage contains two critical points. Then $s$ maps its four critical points bijectively to $V_{s}$, and so $\left|V_{s}\right|=4$.

Let $d=\operatorname{deg}(s)$. By the Riemann-Hurwitz formula $\left|\Omega_{s}\right|=2(d-1)$. Since $\left|V_{s}\right| \leq 3$ and $\operatorname{deg}(s, x)=2$ for every $x \in \Omega_{s}$ it follows that $\left|\Omega_{s}\right| \leq 3(d / 2)$. So $d \leq 4$.

Proposition 3.7. There exist NET maps with constant pullback map that do not satisfy McMullen's constant conditions.

Proof. Let $f$ be an NET map with odd degree whose pullback map is constant. If $f$ satisfies McMullen's constant conditions (see Theorem 1.2), then there are two orientation-preserving branched covering maps $g$ and $s$ and a set $A$ such that $f=g \circ s,|A| \leq 3$, and $V_{s} \subseteq A$. Since $f$ is nearly Euclidean, $\operatorname{deg}(s ; x)=2$ for every $x \in \Omega_{s}$. By Lemma 3.6, either $\operatorname{deg}(s)=2 \operatorname{or} \operatorname{deg}(s)=4$. This is impossible because $\operatorname{deg}(f)$ is an odd number.

Example 3.8. We construct an expanding rational NET map with degree 4 whose Teichmüller map is constant. Let $g$ be the Lattés map described in Example 10.7 of [4]. The critical points of $g$ are $E_{1}, E_{1}^{\prime}, E_{2}, E_{2}^{\prime}, E_{3}, E_{3}^{\prime}$ and the postcritical set of $g$ is $\left\{e_{1}, e_{2}, e_{3}, \infty\right\}$. Moreover, $g\left(e_{i}\right)=g(\infty)=\infty$ and $g\left(E_{i}\right)=g\left(E_{i}^{\prime}\right)=e_{i}$ for $i \in\{1,2,3\}$. Now let $h: \mathbb{P}^{1} \rightarrow \mathbb{P}^{1}$ be an orientation-preserving homeomorphism such that $h\left(e_{1}\right)=e_{1}, h\left(e_{2}\right)=E_{1}, h\left(e_{3}\right)=E_{1}^{\prime}, h(\infty)=\infty$.

Following the description given in Example 10.7 of [4], one sees that $f=h \circ g$ is a NET map whose Teichmüller map is constant so it is combinatorially equivalent to a rational map, $R$. One easily verifies that $R\left(E_{1}\right)=R\left(E_{1}^{\prime}\right)=e_{1}$ mapping with degree $2, R\left(E_{2}\right)=R\left(E_{2}^{\prime}\right)=E_{1}$ mapping with degree $2, R\left(E_{3}\right)=R\left(E_{3}^{\prime}\right)=E_{1}^{\prime}$ mapping with degree 2 , and $R\left(e_{1}\right)=R(\infty)=\infty$ mapping with degree 1 . So $R$ is a rational map without periodic critical points. Let $\mu$ be the Mobiüs transformation that satisfies $\mu\left(E_{1}\right)=0, \mu\left(E_{1}^{\prime}\right)=\infty, \mu\left(e_{1}\right)=1$, and let $F=\mu \circ R \circ \mu^{-1}$. Set $\alpha=\mu\left(E_{2}\right), \beta=\mu\left(E_{2}^{\prime}\right), \gamma=\mu\left(E_{3}\right), \lambda=\mu\left(E_{3}^{\prime}\right)$. Using the branching data of $F$, we have that

$$
F(z)=\frac{(z-\alpha)^{2}(z-\beta)^{2}}{(z-\gamma)^{2}(z-\lambda)^{2}}
$$

The numerator of $F(z)-1$ is given by $(z-\alpha)^{2}(z-\beta)^{2}-(z-\gamma)^{2}(z-\lambda)^{2}$. Since $z=0$ is the unique zero of the numerator of $F(z)-1$ and has multiplicity 2 , it follows that $(z-\alpha)(z-\beta)+(z-\gamma)(z-\lambda)=2 z^{2}$ and that $(z-\alpha)(z-\beta)-(z-\gamma)(z-\lambda)$ is a nonzero constant. This leads to the equations

$$
\begin{gathered}
-(\alpha+\beta)+(\gamma+\lambda)=0, \\
-(\alpha+\beta)-(\gamma+\lambda)=0, \\
\alpha \beta+\gamma \lambda=0 .
\end{gathered}
$$

This forces $\alpha+\beta=0$ and $\gamma+\lambda=0$. So $\alpha=-\beta, \gamma=-\lambda$, and $-\beta^{2}-\lambda^{2}=0$. Hence, $F(z)=\left(z^{2}-\beta^{2}\right)^{2} /\left(z^{2}+\beta^{2}\right)^{2}$ with the restriction $F(F(1))=F(1)$. This restriction implies that either

$$
\frac{(F(1))^{2}-\beta^{2}}{(F(1))^{2}+\beta^{2}}=\frac{1-\beta^{2}}{1+\beta^{2}} \quad \text { or } \quad \frac{(F(1))^{2}-\beta^{2}}{(F(1))^{2}+\beta^{2}}=-\frac{1-\beta^{2}}{1+\beta^{2}} .
$$

Thus, either $(F(1))^{2}=1$ or $(F(1))^{2}=\beta^{4}$. If $(F(1))^{2}=1$, then $F(1)=-1$, and so $\beta^{2}= \pm i$. If $(F(1))^{2}=\beta^{4}$, then $\left(1-\beta^{2}\right)^{4}=\left(1+\beta^{2}\right)^{4} \beta^{4}$. 
We now show that if $\beta^{2}=i$, then the map $F(z)=\left(z^{2}-i\right)^{2} /\left(z^{2}+i\right)^{2}$ is an expanding rational (see [3]) NET map with a constant pullback map. To see this, it suffices to consider $g(w)=(w-i)^{2} /(w+i)^{2}, s(z)=z^{2}$, and $A=\{0,1, \infty\}$. Note that $F=g \circ s$ and $g, s$ and $A$ verify McMullen's constant conditions.

Remark 3.9. The preceding example suggests the following family of rational maps: $F_{n}(z)=\left(z^{n}-i\right)^{2} /\left(z^{n}+i\right)^{2}$, with $n \geq 2$. Each $F_{n}$ has no periodic critical points, so each $F_{n}$ is expanding. Also, note that $F_{n}=g \circ s_{n}$ where $g(w)=(w-i)^{2} /(w+i)^{2}$ and $s_{n}(z)=z^{n}$. If $n$ is even and $A=\{0,1, \infty\}$, then $g, s_{n}$, and $A$ verify McMullen's constant conditions. Thus, if $n$ is even, $F_{n}$ has a constant Teichmüller map.

Example 3.10. We construct a NET map of degree 9 whose Teichmüller map is constant. First of all, we construct an Abelian group of degree $4 \cdot 9=36$ which contains a nonseparating subset. We take $A=\mathbb{Z} / 6 \mathbb{Z} \oplus \mathbb{Z} / 6 \mathbb{Z}$. The 3 -torsion subgroup of $A$ is isomorphic to $\mathbb{Z} / 3 \mathbb{Z} \oplus \mathbb{Z} / 3 \mathbb{Z}$. Example 2.1 and Lemma 2.4 imply that $H=\{ \pm(0,2), \pm(2,2), \pm(2,4), \pm(2,0)\}$ is a nonseparating subset of $A$.

Let $\Lambda_{2}=\mathbb{Z}^{2}$, and let $\Lambda_{1}=3 \Lambda_{2}$. So $\Lambda_{2} / 2 \Lambda_{1} \cong A$. Let $\Gamma_{j}$ be the group generated by rotations of order 2 about elements of $\Lambda_{j}$. Note that $S_{j}^{2}:=\mathbb{R}^{2} / \Gamma_{j}$ is a sphere and that the identity map $i: \mathbb{R}^{2} \rightarrow \mathbb{R}^{2}$ induces a quotient map $\bar{i}: S_{1}^{2} \rightarrow S_{2}^{2}$. This quotient map is a branched covering map of degree $d=\left[\Lambda_{2}: \Lambda_{1}\right]=9$. Let $\Phi:\left(\mathbb{R}^{2}, \Lambda_{2}\right) \rightarrow\left(\mathbb{R}^{2}, \Lambda_{1}\right)$ be the affine map $\Phi(x, y)=(3 x, 3 y)$. The map $\Phi$ induces a homeomorphism $\phi$ from $S_{2}^{2}$ to $S_{1}^{2}$. Then we have the following commutative diagram:

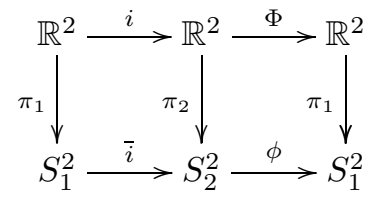

Below is a fundamental domain for the action of $\Gamma_{1}$ on $\mathbb{R}^{2}$. The dots are elements of $\Lambda_{2}$. The lower left corner is $(0,0)$. Points are labeled (bold) by their images in $S_{1}^{2}$ under the map $\pi_{1}$. The map $g:=\phi \circ \bar{i}$ is a Euclidean map with postcritical set $P_{g}=\pi_{1}\left(\Lambda_{1}\right)=\{a, b, c, d\}$. Let $T_{1}:=\mathbb{R}^{2} / 2 \Lambda_{1}$, and let $p_{1}: T_{1} \rightarrow S_{1}^{2}$ be the map defined by $(x, y)+2 \Lambda_{1} \mapsto \pi_{1}(x, y)$. We identify $A$ with $\Lambda_{2} / 2 \Lambda_{1}$ so that the subset $H=\left\{ \pm(0,2)+2 \Lambda_{1}, \pm(2,2)+2 \Lambda_{1}, \pm(2,4)+2 \Lambda_{1}, \pm(2,0)+2 \Lambda_{1}\right\}$ is nonseparating in $\Lambda_{2} / 2 \Lambda_{1}$.

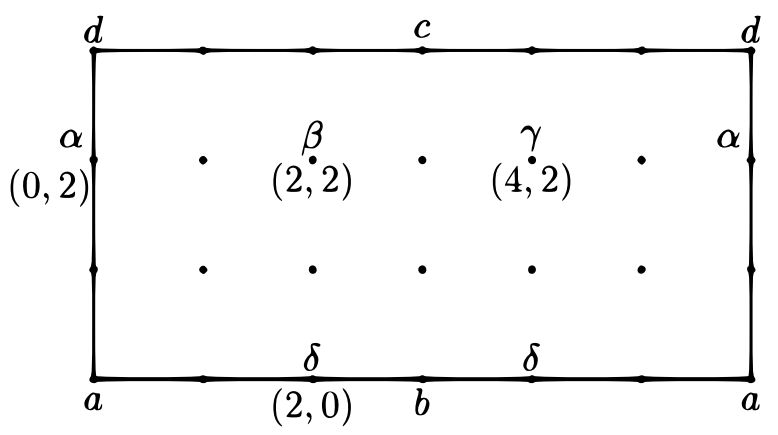

Figure 1. A fundamental domain for the action of $\Gamma_{1}$ on $\mathbb{R}^{2}$. 
Let $h: S_{1}^{2} \rightarrow S_{1}^{2}$ be an orientation-preserving homeomorphism so that $h(a)=\delta$, $h(b)=\beta, h(c)=\alpha, h(d)=\gamma$. Let $f:=h \circ g$. Then $P_{f}=\{\alpha, \beta, \gamma, \delta\}$ and $P_{f}=h\left(P_{g}\right)=p_{1}(H)$, where $H$ is the nonseparating set (identification) contained in $T_{1}$. By Theorem 3.5, the map $f$ is a NET map of degree 9 whose Teichmüller map is constant; however, because of Proposition 3.7, this example does not satisfy McMullen's constant conditions.

\section{NoneXistence RESUlts}

The following nonexistence results can be found in Section 10 of [4].

Theorem 4.1. There does not exist a NET map with degree 2 whose Teichmüller map is constant.

Theorem 4.2. Let $A$ be a finite Abelian group such that $A / 2 A \cong \mathbb{Z} / 2 \mathbb{Z} \oplus \mathbb{Z} / 2 \mathbb{Z}$ and $2 A$ is a cyclic group with odd order. Then $A$ does not contain a nonseparating subset.

Theorem 4.3. There does not exist a NET map with degree an odd square-free integer and constant Teichmüller map.

Our main theorem is a nonexistence result. We begin with the following lemma.

Lemma 4.4. Let $N=p_{1}^{k_{1}} \cdots p_{n}^{k_{n}}$ with $p_{i} \geq 13$. Let $A=\mathbb{Z} / a \mathbb{Z} \oplus \mathbb{Z} /$ b $\mathbb{Z}$ such that $a \mid b, N=a b$, and $a>1$. If $D \subseteq A$ so that $|D| \leq 12$, then there exists a cyclic subgroup $G$ of $A$ such that $G \cap D \subseteq\{0\}$ and $A / G$ is cyclic.

Proof. Since $a \mid b$ and $N=a b$, then $a=p_{1}^{s_{1}} \cdots p_{n}^{s_{n}}$ and $b=p_{1}^{k_{1}-s_{1}} \cdots p_{n}^{k_{n}-s_{n}}$, where $0 \leq 2 s_{i} \leq k_{i}$. Let $I=\{1, \ldots, n\}$, and define $I_{1}:=\left\{i \in I: s_{i}=0\right\}$ and $I_{2}:=I \backslash I_{1}$. Since $a>1, I_{2}$ cannot be empty. Then $A \cong C \oplus P$, where $C=\bigoplus_{i \in I_{1}} \mathbb{Z} / p_{i}^{k_{i}} \mathbb{Z}$ and $P=\bigoplus_{i \in I_{2}}\left(\mathbb{Z} / p_{i}^{s_{i}} \mathbb{Z} \oplus \mathbb{Z} / p_{i}^{k_{i}-s_{i}} \mathbb{Z}\right)$. Without loss of generality we may assume that $A=C \oplus P$. Now, for each $i \in I_{2}$ let $\phi_{i}: A \rightarrow \mathbb{Z} / p_{i}^{s_{i}} \mathbb{Z} \oplus \mathbb{Z} / p_{i}^{k_{i}-s_{i}} \mathbb{Z}$ be the canonical projection. Since $\left|\phi_{i}(D)\right| \leq 12$ and $p_{i} \geq 13$, there exists a cyclic subgroup $G_{i}$ of $\mathbb{Z} / p_{i}^{s_{i}} \mathbb{Z} \oplus \mathbb{Z} / p_{i}^{k_{i}-s_{i}} \mathbb{Z}$ such that $G_{i} \cap \phi_{i}(D) \subseteq\{0\}$ and $\left(\mathbb{Z} / p_{i}^{s_{i}} \mathbb{Z} \oplus \mathbb{Z} / p_{i}^{k_{i}-s_{i}} \mathbb{Z}\right) / G_{i}$ is cyclic. The subgroup $G:=\bigoplus_{i \in I_{2}} G_{i}$ satisfies the conclusion.

Theorem 4.5. Let $A$ be a finite Abelian group generated by two elements such that $A / 2 A \cong \mathbb{Z} / 2 \mathbb{Z} \oplus \mathbb{Z} / 2 \mathbb{Z}$. If $|A|=4 p_{1}^{k_{1}} p_{2}^{k_{2}} \cdots p_{n}^{k_{n}}$ with $p_{i}$ prime, $p_{i} \geq 13$, and $|k|=k_{1}+k_{2}+\cdots+k_{n} \geq 1$, then $A$ does not contain a nonseparating subset.

Proof. We proceed by induction on $|k|$. If $|k|=1$, then $A \cong \mathbb{Z} / 2 \mathbb{Z} \oplus \mathbb{Z} / 2 p \mathbb{Z}$. Since $2 A$ is cyclic with odd order, by Theorem 4.2 the conclusion follows. Now, suppose the conclusion holds for any $|k| \in\{1, \ldots, m-1\}$ and assume that $|A|=4 p_{1}^{k_{1}} p_{2}^{k_{2}} \cdots p_{n}^{k_{n}}$, where $k_{1}+\cdots+k_{n}=m$. Then there are two positive odd integers $a$ and $b$ such that $A \cong \mathbb{Z} / 2 a \mathbb{Z} \oplus \mathbb{Z} / 2 b \mathbb{Z},|A|=4 a b$, and $a$ divides $b$. If $a=1$, then $2 A$ is a cyclic group with odd order. By Theorem 4.2 the conclusion follows. Now, assume that $A=\mathbb{Z} / 2 \mathbb{Z} \oplus \mathbb{Z} / 2 \mathbb{Z} \oplus \mathbb{Z} / a \mathbb{Z} \oplus \mathbb{Z} / b \mathbb{Z}$ with $a>1$ and proceed by contradiction. Suppose that $A$ contains a nonseparating subset $H=H_{1} \amalg H_{2} \amalg H_{3} \amalg H_{4}$, where each $H_{i}=\left\{ \pm h_{i}\right\}$. Then, either $\mathbb{Z} / a \mathbb{Z} \oplus \mathbb{Z} / b \mathbb{Z} \subseteq\langle H\rangle$ or $(\mathbb{Z} / a \mathbb{Z} \oplus \mathbb{Z} / b \mathbb{Z}) \cap(A \backslash\langle H\rangle) \neq \emptyset$.

Case I. $\mathbb{Z} / a \mathbb{Z} \oplus \mathbb{Z} / b \mathbb{Z} \subseteq\langle H\rangle$. Let $\phi: A \rightarrow \mathbb{Z} / a \mathbb{Z} \oplus \mathbb{Z} / b \mathbb{Z}$ be the canonical projection, and let $D:=\left\{\phi\left(h_{i}\right) \pm \phi\left(h_{j}\right): i, j \in\{1,2,3,4\}\right.$ and $\left.i<j\right\}$. The cardinality of $D$ is at most 12 . By Lemma 4.4 there exists a cyclic subgroup $G$ of $\mathbb{Z} / a \mathbb{Z} \oplus \mathbb{Z} / b \mathbb{Z}$ such 
that $G \cap D \subseteq\{0\}$ and $(\mathbb{Z} / a \mathbb{Z} \oplus \mathbb{Z} / b \mathbb{Z}) / G$ is cyclic. Then, by Lemma 2.10, we may, and do, assume that $\left\langle\phi\left(h_{1}\right), \phi\left(h_{2}\right)\right\rangle=\mathbb{Z} / a \mathbb{Z} \oplus \mathbb{Z} / b \mathbb{Z}$ and $h_{2}, h_{3}$, and $h_{4}$ all differ by an element of order 2 .

If $a=b$, there exists a cyclic subgroup $B$ of $A$ of order $2 b$ such that $h_{2} \in B$ and $A / B$ is cyclic. Since $h_{2}, h_{3}$, and $h_{4}$ all differ by an element of order 2 , then $h_{2}+B$, $h_{3}+B, h_{4}+B$ lie in the subgroup of order 2 in $A / B$. They are not all equal because $B$ does not contain three elements of order 2. So either $c_{1}=c_{2}=0$ and $c_{4}=b$ or $c_{1}=0$ and $c_{3}=c_{4}=b$. Thus, the order of $h_{1}+B$ divides 2. Hence $2 \phi\left(h_{1}\right) \in B$, and so $\phi\left(h_{1}\right)=((b+1) / 2)\left(2 \phi\left(h_{1}\right)\right) \in B$. Therefore $\left\langle\phi\left(h_{1}\right), \phi\left(h_{2}\right)\right\rangle \subseteq B$. This is a contradiction because $B$ is cyclic and $\left\langle\phi\left(h_{1}\right), \phi\left(h_{2}\right)\right\rangle=\mathbb{Z} / a \mathbb{Z} \oplus \mathbb{Z} / b \mathbb{Z}$.

If $a<b$, set $\tilde{A}:=\mathbb{Z} / 2 \mathbb{Z} \oplus \mathbb{Z} / 2 \mathbb{Z} \oplus \mathbb{Z} / b \mathbb{Z} \oplus \mathbb{Z} / b \mathbb{Z}$ and let $i_{c}$ be the canonical monomorphism $i_{c}: A \rightarrow \tilde{A}$ defined by $i_{c}(x, y, z, t)=(x, y,(b / a) z, t)$. By Lemma 2.4 $i_{c}(H)$ is a nonseparating subset of $\tilde{A}$. Now, set $\mu:=i_{c}\left(\phi\left(h_{1}\right)\right)$ and $\nu:=i_{c}\left(\phi\left(h_{2}\right)\right)$. Since $\left\langle\phi\left(h_{1}\right), \phi\left(h_{2}\right)\right\rangle=\mathbb{Z} / a \mathbb{Z} \oplus \mathbb{Z} / b \mathbb{Z}$, then $\langle\mu, \nu\rangle=i_{c}(\mathbb{Z} / a \mathbb{Z} \oplus \mathbb{Z} / b \mathbb{Z})=\langle b / a\rangle \oplus \mathbb{Z} / b \mathbb{Z}$. Furthermore, $i_{c}\left(h_{2}\right), i_{c}\left(h_{3}\right)$, and $i_{c}\left(h_{4}\right)$ all differ by an element of order 2 . Since $\nu \in \mathbb{Z} / b \mathbb{Z} \oplus \mathbb{Z} / b \mathbb{Z}$ there exists a cyclic subgroup $\tilde{B}$ of $\tilde{A}$ of order $2 b$ such that $i_{c}\left(h_{2}\right) \in \tilde{B}$ and $\tilde{A} / \tilde{B}$ is cyclic. Then $i_{c}\left(h_{2}\right)+\tilde{B}, i_{c}\left(h_{3}\right)+\tilde{B}, i_{c}\left(h_{4}\right)+\tilde{B}$ lie in the subgroup of order 2 in $\tilde{A} / \tilde{B}$. They are not all equal because $\tilde{B}$ does not contain three elements of order 2. So either $c_{1}=c_{2}=0$ and $c_{4}=b$ or $c_{1}=0$ and $c_{3}=c_{4}=b$. Thus, $i_{c}\left(h_{1}\right)+\tilde{B}$ must have order 2 in $\tilde{A} / \tilde{B}$. Hence $2 \mu \in \tilde{B}$, and so $\mu=((b+1) / 2)(2 \mu) \in \tilde{B}$. Therefore $\langle\mu, \nu\rangle \subseteq \tilde{B}$. This is a contradiction because $\tilde{B}$ is cyclic and $\langle\mu, \nu\rangle=\langle b / a\rangle \oplus \mathbb{Z} / b \mathbb{Z}$.

Case II. $(\mathbb{Z} / a \mathbb{Z} \oplus \mathbb{Z} / b \mathbb{Z}) \cap(A \backslash\langle H\rangle) \neq \emptyset$. This means that $\langle H\rangle$ does not contain a copy of $\mathbb{Z} / a \mathbb{Z} \oplus \mathbb{Z} / b \mathbb{Z}$. Then, $H \subset\langle H\rangle \subseteq A^{\prime}$ where $A^{\prime}$ is a proper subgroup of $A$ whose order has the form $4 r$. Obviously, $r$ divides $|A| / 4$ and $r<|A| / 4$. Since $A^{\prime}$ is a finite Abelian group generated by 2 elements, we can apply the inductive hypothesis to $A^{\prime}$ and conclude that $A^{\prime}$ does not contain a nonseparating subset. However, this contradicts Lemma 2.6.

This proves Theorem 4.5.

As an immediate consequence of Theorem 4.5, we have the following corollary.

Corollary 4.6. Let $n=p_{1}^{k_{1}} p_{2}^{k_{2}} \cdots p_{n}^{k_{n}}$ with $p_{i}$ prime, $p_{i} \geq 13$. There does not exist a NET map with degree $n$ whose Teichmüller map is constant.

We proved in 8 the following theorems. Their proofs are similar to the proof of Theorem 4.5] For further details see Chapter 5 of 8 .

Theorem 4.7. Let $A$ be a finite Abelian group generated by two elements such that $A / 2 A \cong \mathbb{Z} / 2 \mathbb{Z} \oplus \mathbb{Z} / 2 \mathbb{Z}$. If $|A|=4 p^{2}$ with $p$ prime and $p \geq 5$, then $A$ does not contain a nonseparating subset.

Theorem 4.8. Let $A$ be a finite Abelian group generated by two elements such that $A / 2 A \cong \mathbb{Z} / 2 \mathbb{Z} \oplus \mathbb{Z} / 2 \mathbb{Z}$. If $|A|=4 p^{3}$ with $p$ prime and $p \geq 7$, then $A$ does not contain a nonseparating subset.

As immediate consequences, we have the following corollaries.

Corollary 4.9. For any prime $p \geq 5$ there does not exist a NET map with degree $p^{2}$ whose Teichmüller map is constant. 
Corollary 4.10. For any prime $p \geq 7$ there does not exist a NET map with degree $p^{3}$ whose Teichmüller map is constant.

Corollaries 4.6, 4.9, and 4.10 strongly support the conjecture made by J. Cannon et al. in Section 10 of [4. This conjecture has been verified by computer for all NET maps with degree at most 300. See section 2 of [6] for further progress in this direction.

Conjecture. There exists a NET map with degree $d$ and constant Teichmüller map if and only if $d>2$ and $d$ is divisible by either 2 or 9 .

\section{ON Thurston MAPs OF DEgReE 2}

Let $f: S^{2} \rightarrow S^{2}$ be a Thurston map, and let $P_{f}$ be its postcritical set. Combining statements 1 and 4 of Theorem 5.1 of [2] implies that the pullback map $\Sigma_{f}$ is constant if and only if for every essential simple closed curve $\alpha$ in $S^{2} \backslash P_{f}$, every connected component of $f^{-1}(\alpha)$ is either trivial or peripheral in $S^{2} \backslash P_{f}$. We use this result to conclude that there does not exist a Thurston map of degree 2 with at least four postcritical points whose Teichmüller map is constant.

A branched covering map $f: S^{2} \rightarrow S^{2}$ is said to be a topological polynomial if there exists a critical point $w$ such that $f^{-1}(w)=\{w\}$. If $f$ is a Thurston polynomial and $\left|P_{f}\right|>2$, there is a unique point $w$ such that $f^{-1}(w)=\{w\}$; we call this point $\infty$.

Lemma 5.1. Let $f: S^{2} \rightarrow S^{2}$ be a branched covering map of degree $n$. Suppose that $\operatorname{deg}(f, w)=n$ for some $w \in S^{2}$. If $U \subset S^{2}$ is homeomorphic to an open disk with $f(w) \notin U$, then every component of $f^{-1}(U)$ is homeomorphic to a disk.

Proof. Straightforward consequence of Lemma 5.1 of [1].

Theorem 5.2. Let $f: S^{2} \rightarrow S^{2}$ be a Thurston map of degree $n$ with $\left|P_{f}\right|=m$. Suppose there exists $w \in S^{2}$ such that $\operatorname{deg}(f, w)=n$. Suppose additionally that there exists $c \in P_{f} \backslash V_{f}$, and let $k=\left|f^{-1}(c) \cap P_{f}\right|$.

(1) If $w \in P_{f}$ and $m-k \geq 3$, then $\Sigma_{f}$ cannot be constant.

(2) If $w \notin P_{f}, k \geq 2$, and $m-k \geq 2$, then $\Sigma_{f}$ cannot be constant.

Proof. To prove statement (1), let $\alpha$ be a simple closed curve contained in $S^{2} \backslash P_{f}$ that separates the points $c$ and $f(w)$ from the other postcritical points of $f$. Let $D$ be the connected component of $S^{2} \backslash \alpha$ that contains the points $c$ and $f(w)$. By Lemma [5.1, $f^{-1}(D)$ is connected. Furthermore, $\left.f\right|_{f^{-1}(D)}: f^{-1}(D) \rightarrow D$ is a branched covering map of degree $n$ with exactly one critical point. It follows that $f^{-1}(D) \cap P_{f}=\{w\} \cup\left(f^{-1}(c) \cap P_{f}\right)$. So, one connected component of $S^{2} \backslash f^{-1}(\alpha)$ contains exactly $k+1$ postcritical points of $f$. Then the other connected component of $S^{2} \backslash f^{-1}(\alpha)$ contains $m-(k+1)$ postcritical points of $f$. Since $m-(k+1) \geq 2$, $f^{-1}(\alpha)$ is essential in $S^{2} \backslash P_{f}$. This proves statement (1) of Theorem 5.2 .

To prove statement (2), let $\alpha$ and $D$ be as in the previous paragraph. Since $w \notin P_{f}, f^{-1}(D) \cap P_{f}=f^{-1}(c) \cap P_{f}$. Under the assumptions $k \geq 2$ and $m-k \geq 2$, it follows that $f^{-1}(\alpha)$ is essential in $S^{2} \backslash P_{f}$.

This proves Theorem 5.2 .

Corollary 5.3. Let $f$ be a Thurston map of degree 2. If $\left|P_{f}\right| \geq 4$, then $\Sigma_{f}$ cannot be constant. 
Proof. Let $f$ be a Thurston map of degree 2. We proceed by cases:

- $\left|P_{f}\right|=4$. If $f$ is a Euclidean Thurston map, $\mathcal{T}_{f}$ can be identified with the upper half plane $\mathbb{H}$ and $\Sigma_{f}: \mathbb{H} \rightarrow \mathbb{H}$ is given by $\Sigma_{f}(z)=(d z+b) /(c z+a)$ where $a, b, c, d \in \mathbb{Z}$ and $a d-b c=\operatorname{deg}(f)$. So $\Sigma_{f}$ cannot be constant.

We now assume that $f$ is not a Euclidean Thurston map. In this case, $P_{f} \cap C_{f} \neq \emptyset$. The set $P_{f} \backslash V_{f}$ contains exactly two elements, say $a$ and $b$. If $\left|f^{-1}(a) \cap P_{f}\right|=\left|f^{-1}(b) \cap P_{f}\right|=2$, then $P_{f}=f^{-1}(a) \amalg f^{-1}(b)$ and no critical point would be postcritical. Hence $\left|f^{-1}(a) \cap P_{f}\right|=1$ or $\left|f^{-1}(b) \cap P_{f}\right|=1$. So there exist $w \in P_{f}$ with $\operatorname{deg}(f, w)=2$ and $c \in P_{f} \backslash V_{f}$ with $1=\left|f^{-1}(c) \cap P_{f}\right|$. Part (1) of Theorem 5.2 implies that $\Sigma_{f}$ cannot be constant.

- $\left|P_{f}\right| \geq 5$. If $f$ is a topological polynomial, then $\infty \in P_{f}$ and $\operatorname{deg}(f, \infty)=2$. Let $x \in \Omega_{f} \backslash\{\infty\}$ and let $\mathcal{O}(x)$ be the forward orbit of $x$ under $f$. Since $4 \leq|\mathcal{O}(x)|<\infty$, there exists $c \in \mathcal{O}(x)$ such that $\left|f^{-1}(c) \cap P_{f}\right|=1$. By part (1) of Theorem 5.2 (take $w=\infty$ ) it follows that $\Sigma_{f}$ cannot be constant.

We now assume that $f$ is not a topological polynomial. So $f$ has two critical points, say $a$ and $b$, and neither of them is a fixed point. Let $\mathcal{O}(a)$ and $\mathcal{O}(b)$ be the forward orbits of $a$ and $b$, respectively. If one of these critical points, say $a$, is preperiodic, then there exists $c \in \mathcal{O}(a)$ such that $\left|f^{-1}(c) \cap P_{f}\right|=2$. In this case, take $w=a$ and apply part (2) of Theorem 5.2 to conclude that $\Sigma_{f}$ cannot be constant. If $a$ and $b$ are both periodic, then $a \notin V_{f}$ or $b \notin V_{f}$. Without loss of generality we may assume that $b \notin V_{f}$. Then $1=\left|f^{-1}(b) \cap P_{f}\right|$. In this case, take $w=a$ and apply part (1) of Theorem 5.2 to conclude that $\Sigma_{f}$ cannot be constant.

Remark 5.4. In Proposition 5.3 of [7], Koch shows that if $f$ is a topological polynomial with $\left|\Omega_{f}\right|=2$, then $X: \mathcal{W}_{f} \rightarrow \mathcal{M}_{P_{f}}$ is injective. So a moduli space map $g_{f}$ exists. This implies that $\Sigma_{f}$ cannot be constant. For further details, see Proposition 5.3 and Corollary 5.4 of [7].

\section{Appendix A. Group theOrY}

Proposition A.1. Let $G$ be a finite cyclic group of order $n$, and let $h$ be an element of order $m$ in $G$. Then there exists $g \in G$ such that $\langle g\rangle=G$ and $g^{n / m}=h$.

Proof. Choose $a \in G$ such that $\langle a\rangle=G$. Then $\left\langle a^{n / m}\right\rangle=\langle h\rangle$, so there exists $r \in \mathbb{N}$ such that $a^{n r / m}=h$; of course $\operatorname{gcd}(r, m)=1$. Also, if $d \in \mathbb{N}$, then $a^{n d / m}=h$ if and only if $d \equiv r \bmod m$.

Let $q$ be the product of the primes which divide $n / m$ but do not divide $m$. Thus, $\operatorname{gcd}(m, q)=1$ and $\operatorname{gcd}(s, n)=1$ if and only if $\operatorname{gcd}(s, m)=\operatorname{gcd}(s, q)=1$. By the Chinese remainder theorem, we may choose $\tau \in \mathbb{N}$ such that $\tau \equiv r \bmod m$ and $\tau \equiv 1 \bmod q$. Then $\operatorname{gcd}(\tau, n)=1$, so $a^{\tau}$ generates $G$. Also $\left(a^{\tau}\right)^{n / m}=h$, as required.

Proposition A.2. Every element of $\mathbb{Z} / n \mathbb{Z} \oplus \mathbb{Z} / n \mathbb{Z}$ is a multiple of a basis element.

Proof. In fact, let $g=(\bar{x}, \bar{y}) \in \mathbb{Z} / n \mathbb{Z} \oplus \mathbb{Z} / n \mathbb{Z}$. If $\bar{x}=\overline{0}$ or $\bar{y}=\overline{0}$, the proposition follows. Assume $\bar{x} \neq \overline{0}$ and $\bar{y} \neq \overline{0}$; then $g=d(\overline{x / d}, \overline{y / d})$ where $d=\operatorname{gcd}(x, y)$. Since $\operatorname{gcd}(x / d, y / d)=1,(\overline{x / d}, \overline{y / d})$ is a basis element and the proposition follows.

Proposition A.3. Assume that $A=\mathbb{Z} / n \mathbb{Z} \oplus \mathbb{Z} / n \mathbb{Z}$. Let $A^{\prime}$ be a subgroup of $A$, and let $B^{\prime}$ be a cyclic subgroup of $A^{\prime}$ so that $A^{\prime} / B^{\prime}$ is also cyclic. Then there exists a cyclic subgroup $B$ of $A$ such that $A / B$ is cyclic and $A^{\prime} \cap B=B^{\prime}$. 
Proof. Let $S_{1}, \ldots, S_{\ell}$ be the Sylow subgroups of $A$. Since every subgroup $C$ of $A$ is the direct sum of $C \cap S_{1}, \ldots, C \cap S_{\ell}$, it suffices to prove the proposition for $p$-groups. So we may, and do, assume that $n=p^{r}$ where $p$ is a prime number and $r \in \mathbb{Z}^{+}$.

Case I. Suppose that $A^{\prime}$ is cyclic. By Proposition A.2, every element of $A^{\prime}$ is a multiple of a basis element of $A$. Let $v \in A$ be a basis element such that $\langle v\rangle$ contains $A^{\prime}$. Choose $w \in A$ so that $\{v, w\}$ is a basis for $A$. Let $\varphi$ be the automorphism of $A$ defined on these generators by $\varphi(v)=(1,0)$ and $\varphi(w)=(0,1)$. Let $b^{\prime}$ be a generator of $B^{\prime}$, and let $k=o\left(b^{\prime}\right)$. Since $\varphi\left(b^{\prime}\right) \in\langle(1,0)\rangle$, by Proposition A.1. there exists $g \in \mathbb{Z} / n \mathbb{Z}$ such that $\mathbb{Z} / n \mathbb{Z}=\langle g\rangle$ and $\varphi\left(b^{\prime}\right)=(n / k)(g, 0)$. Let $T$ be the automorphism of $A$ defined on generators by $T(g, 0)=(1,0)$ and $T(0,1)=(0,1)$. Now consider the isomorphism $f=T \circ \varphi$. Then $A^{\prime}$ is isomorphic to $f\left(A^{\prime}\right)$ which is a subgroup contained in the subgroup $\langle(1,0)\rangle$. Let $b \in A$ so that $f(b)=(1, k)$. Using these coordinates, we have $\langle f(b)\rangle \cap f\left(A^{\prime}\right)=\langle(n / k, 0)\rangle$. Then $\langle b\rangle \cap A^{\prime}=\left\langle b^{\prime}\right\rangle$. Finally, we set $B=\langle b\rangle$. Since $o(b)=n, A / B$ is cyclic as required.

Case II. Suppose that $A^{\prime}$ is not cyclic. In this case, there are positive integers $s, t$ so that $A^{\prime} \cong \mathbb{Z} / p^{s} \mathbb{Z} \oplus \mathbb{Z} / p^{t} \mathbb{Z}$. We first show that no cyclic subgroup of $A^{\prime}$ properly contains $B^{\prime}$. If $C$ is a cyclic subgroup of $A^{\prime}$ such that $B^{\prime} \subsetneq C$, then $B^{\prime} \subseteq p C$, and so $B^{\prime} \subseteq p A^{\prime} \subsetneq A^{\prime}$. The Third Isomorphism Theorem for groups would then imply that the quotient group $\left(A^{\prime} / B^{\prime}\right) /\left(p A^{\prime} / B^{\prime}\right)$ is isomorphic to $A^{\prime} / p A^{\prime}$. This is a contradiction because $A^{\prime} / B^{\prime}$ is cyclic and $A^{\prime} / p A^{\prime}$ is not. Now let $b^{\prime}$ be a generator of $B^{\prime}$, and let $b$ be a basis element of $A$ such that $\langle b\rangle$ contains $B^{\prime}$. Set $B=\langle b\rangle$. Because $b$ is a basis element of $A, A / B$ is cyclic. Finally, since $A^{\prime} \cap B$ is a cyclic subgroup of $A^{\prime}$ that contains $B^{\prime}$, we have $A^{\prime} \cap B=B^{\prime}$.

Proposition A.4. Let $A$ be a finite Abelian group generated by two elements. Let $A^{\prime}$ be a subgroup of $A$, and let $B^{\prime}$ be a cyclic subgroup of $A^{\prime}$ so that $A^{\prime} / B^{\prime}$ is also cyclic. Then there exists a cyclic subgroup $B$ of $A$ such that $A / B$ is cyclic and $A^{\prime} \cap B=B^{\prime}$.

Proof. We may assume that $A=\mathbb{Z} / m \mathbb{Z} \oplus \mathbb{Z} / n \mathbb{Z}$ where $m$ and $n$ are positive integers such that $m \mid n$. Let $i_{c}: A \rightarrow \mathbb{Z} / n \mathbb{Z} \oplus \mathbb{Z} / n \mathbb{Z}$ be the canonical monomorphism defined on generators by $i_{c}(1,0)=(n / m, 0)$ and $i_{c}(0,1)=(0,1)$. Then $A$ is isomorphic to the subgroup $\langle n / m\rangle \oplus \mathbb{Z} / n \mathbb{Z}$. So, without loss of generality, we may assume that $A$ is a subgroup of $\mathbb{Z} / n \mathbb{Z} \oplus \mathbb{Z} / n \mathbb{Z}$. Now let $A^{\prime}$ be a subgroup of $A$, and let $B^{\prime}$ be a cyclic subgroup of $A^{\prime}$ so that $A^{\prime} / B^{\prime}$ is cyclic. By Proposition A.3, there exists a cyclic subgroup $B$ of $\mathbb{Z} / n \mathbb{Z} \oplus \mathbb{Z} / n \mathbb{Z}$ such that $(\mathbb{Z} / n \mathbb{Z} \oplus \mathbb{Z} / n \mathbb{Z}) / B$ is cyclic and $A^{\prime} \cap B=B^{\prime}$. Now, set $\tilde{B}=A \cap B$. Obviously $\tilde{B}$ is a cyclic subgroup of $A$ and $A^{\prime} \cap \tilde{B}=A^{\prime} \cap(A \cap B)=\left(A^{\prime} \cap A\right) \cap B=A^{\prime} \cap B=B^{\prime}$. Finally, using the canonical projection $\phi: A \rightarrow(\mathbb{Z} / n \mathbb{Z} \oplus \mathbb{Z} / n \mathbb{Z}) / B$ defined by $x \mapsto x+B$, one sees that $\operatorname{Ker}(\phi)=A \cap B=\tilde{B}$, and so $A / \tilde{B}$ is isomorphic to $\phi(A)$. Since $(\mathbb{Z} / n \mathbb{Z} \oplus \mathbb{Z} / n \mathbb{Z}) / B$ is cyclic, $\phi(A)$ is also, and therefore $A / \tilde{B}$ is cyclic.

Appendix B. Classifying nonseparating subsets of $\mathbb{Z} / 4 \mathbb{Z} \oplus \mathbb{Z} / 4 \mathbb{Z}$

Theorem B.1. There are exactly three Hurwitz classes of nonseparating subsets in $A=\mathbb{Z} / 4 \mathbb{Z} \oplus \mathbb{Z} / 4 \mathbb{Z}$. The following subsets are representatives for each class:

$$
\begin{gathered}
H_{1}=\{(0,0), \pm(1,0), \pm(1,2),(2,0)\}, \\
H_{2}=\{ \pm(1,0), \pm(0,1), \pm(2,1), \pm(1,2)\}, \\
H_{3}=\{ \pm(1,0), \pm(0,1), \pm(1,1), \pm(3,1)\} .
\end{gathered}
$$


Proof. It is known that $H_{1}=\{(0,0), \pm(1,0), \pm(1,2),(2,0)\}$ is a nonseparating subset of $A$ (see Example 2.5). We now show that $H_{2}$ is a nonseparating subset of $A$. Let $B$ be a cyclic subgroup of $A$ such that $A / B$ is cyclic. Then $B \cong A / B \cong \mathbb{Z} / 4 \mathbb{Z}$. There are only six possible choices for $B:\langle(1,0)\rangle,\langle(0,1)\rangle,\langle(1,2)\rangle,\langle(2,1)\rangle,\langle(3,1)\rangle$, and $\langle(1,1)\rangle$. If $B=\langle(1,1)\rangle$ or $B=\langle(1,3)\rangle$, one verifies in these cases that $c_{1}=c_{2}=c_{3}=c_{4}=1$. If $B \neq\langle(1,1)\rangle$ and $B \neq\langle(1,3)\rangle$, one verifies in these cases that $c_{1}=0$ and $c_{2}=c_{3}=1$. Similarly, it is not difficult to show that $H_{3}$ is also a nonseparating subset of $A$.

It remains to show that the Hurwitz classes of $H_{1}, H_{2}$, and $H_{3}$ are the only possible Hurwitz classes of nonseparating subsets of $A$.

Let $H$ be a nonseparating subset of $A$. Using the fact that $\mathbb{Z} / 2 \mathbb{Z} \oplus \mathbb{Z} / 2 \mathbb{Z}$ does not contain any nonseparating subset and Lemma 2.6, it follows that $H$ must contain at least one element of order 4 .

We first show that if $H$ contains the point $(0,0)$, then $H$ is in the Hurwitz class of $H_{1}$. Suppose that $(0,0) \in H$. Since $H$ contains at least one element of order 4 , by a group automorphism of $A$, we may assume that $H$ contains $\pm(1,0)$. Let $B=\langle(1,0)\rangle$ and consider the generator $(0,1)+B$ of $A / B$. Since $c_{1}=c_{2}=0$, we must have $c_{3}=0$. So $(2,0) \in H$. Now take $B^{\prime}=\langle(0,1)\rangle$ and consider the generator $(1,0)+B^{\prime}$ of $A / B^{\prime}$. Since $c_{2}^{\prime}=c_{3}^{\prime}=1$, exactly one of the following must be contained in $H:\{ \pm(1,1)\},\{ \pm(1,2)\},\{ \pm(1,3)\}$. Now let $B^{\prime \prime}=\langle(1,2)\rangle$. Since $(0,0),(2,0) \in H$, any generator for $A / B^{\prime \prime}$ leads us to $c_{1}^{\prime \prime}=c_{2}^{\prime \prime}=0$. Then $c_{3}^{\prime \prime}=0$, and so $\pm(1,2) \in H$. Therefore, $H$ would be in the Hurwitz class of $H_{1}$.

If $H$ is a nonseparating subset of $A$ that contains an element of order 2, say $\tau$, then $H^{\prime}=H+\tau$ is a nonseparating subset of $A$ containing the point $(0,0)$. The above argument shows that $H^{\prime}$ is in the Hurwitz class of $H_{1}$.

We now assume that $H$ contains no elements of $\langle 2\rangle \oplus\langle 2\rangle$. Then $H$ cannot be in the Hurwitz class of $H_{1}$. Moreover, $H$ must contain exactly four of the following subsets:

$$
\{ \pm(1,0)\}, \quad\{ \pm(0,1)\}, \quad\{ \pm(1,1)\}, \quad\{ \pm(1,2)\}, \quad\{ \pm(2,1)\}, \quad\{ \pm(1,3)\} .
$$

So $H$ contains a basis of $A$. By a group automorphism of $A$, we may assume that $H$ contains $\pm(1,0)$ and $\pm(0,1)$. Hence $H$ is in the Hurwitz class of at least one of the following subsets:

$$
\begin{array}{ll}
J_{1}=\{ \pm(1,0), \pm(0,1), \pm(1,2), \pm(2,1)\}, & J_{2}=\{ \pm(1,0), \pm(0,1), \pm(1,2), \pm(1,1)\}, \\
J_{3}=\{ \pm(1,0), \pm(0,1), \pm(1,2), \pm(3,1)\}, & J_{4}=\{ \pm(1,0), \pm(0,1), \pm(2,1), \pm(1,1)\}, \\
J_{5}=\{ \pm(1,0), \pm(0,1), \pm(2,1), \pm(3,1)\}, & J_{6}=\{ \pm(1,0), \pm(0,1), \pm(1,1), \pm(3,1)\} .
\end{array}
$$

In this paragraph we show that $J_{2}, \ldots, J_{6}$ are in the same Hurwitz class. In fact, note that $J_{4}+(2,0)=J_{5}$, the automorphism of $A$ defined by $(x, y) \mapsto(y, x)$ maps bijectively $J_{2}$ onto $J_{4}$ and $J_{3}$ onto $J_{5}$; and the automorphism of $A$ defined by $(x, y) \mapsto(x+y, y)$ maps bijectively $J_{5}$ onto $J_{6}$.

To show that either $H$ is in the Hurwitz class of $H_{2}$ or in the Hurwitz class of $H_{3}$, it suffices to show that $J_{1}$ and $J_{6}$ are not in the same Hurwitz class. To do so, we proceed by contradiction. Suppose that there exists an automorphism of $A$, say $\varphi$, and an element $\tau \in A$ with $2 \tau=0$ such that $\varphi\left(J_{1}\right)+\tau=J_{6}$. Regardless of the choice of the automorphism we have $\varphi(1,0) \equiv \varphi(1,2)(\bmod 2), \varphi(0,1) \equiv \varphi(2,1)$ $(\bmod 2)$, and $\varphi(1,0) \not \equiv \varphi(0,1)(\bmod 2)$. So the set $\varphi\left(J_{1}\right)+\tau(\bmod 2)$ has exactly 
two elements. This yields a contradiction because the set $J_{6}(\bmod 2)$ contains exactly three elements.

\section{List of Symbols}

$\mathbb{N}=\{0,1,2, \ldots\}$.

$\mathbb{Z}$ is the set of integer numbers.

$\mathbb{Z}^{+}$is the set of positive integers.

$\mathbb{R}$ is the set of real numbers.

$\mathbb{C}$ is the complex plane.

$\mathbb{P}^{1}$ is the Riemann Sphere.

$\mathbb{Z}^{2}$ is the 2-dimensional integer lattice.

$\Omega_{f}$ is a critical set of the map $f$.

$V_{f}$ is a critical value set of the map $f$.

$P_{f}$ is a postcritical set of the Thurston map $f$.

$\Sigma_{f}$ is the pullback map induced by the Thurston map $f$.

$\operatorname{deg}(f)$ is the degree of the map $f$.

$\operatorname{deg}(f, w)$ is the local degree of $f$ at the point $w$.

$|A|$ is the cardinality of the set $A$.

$o(g)$ is the order of the group element $g$.

\section{ACKNOWLEDGMENTS}

The results described in this paper are part of the author's $\mathrm{PhD}$ thesis under the supervision of William Floyd. The author would like to thank William Floyd for his essential guidance and for his constant help, support and patience. The author also would like to thank Walter Parry for several helpful discussions, useful comments, and for sharing his mathematical insights.

\section{REFERENCES}

[1] B. Bielefeld, Y. Fisher, and J. Hubbard, The classification of preperiodic polynomials as dynamical systems, Journal of the American Mathematical Society, Vol. 5, No. 4, October 1992.

[2] Xavier Buff, Adam Epstein, Sarah Koch, and Kevin Pilgrim, On Thurston's pullback map, Complex dynamics, A K Peters, Wellesley, MA, 2009, pp. 561-583, DOI 10.1201/b10617-20. MR2508269

[3] Mario Bonk and Daniel Meyer, Expanding Thurston maps, Mathematical Surveys and Monographs, vol. 225, American Mathematical Society, Providence, RI, 2017. MR.3727134

[4] J. W. Cannon, W. J. Floyd, W. R. Parry, and K. M. Pilgrim, Nearly Euclidean Thurston maps, Conform. Geom. Dyn. 16 (2012), 209-255 (electronic).

[5] A. Douady and J. H. Hubbard, A proof of Thurston's topological characterization of rational functions, Acta Math. 171 (1993), 263-297.

[6] William Floyd, Gregory Kelsey, Sarah Koch, Russell Lodge, Walter Parry, Kevin M. Pilgrim, and Edgar Saenz, Origami, affine maps, and complex dynamics, Arnold Math. J. 3 (2017), no. 3, 365-395, DOI 10.1007/s40598-017-0071-0. MR3692494

[7] S. Koch, Teichmüller Theory and critically finite endomorphisms, Advances in Mathematics, vol. $248,2013$.

[8] E. A. Saenz, On Nearly Euclidean Thurston maps, Ph.D. Thesis, Virginia Tech, 2012.

Department of Mathematics, Virginia Tech, Blacksburg, Virginia 24061

Email address: easaenzm@math.vt.edu 